\title{
The Role of Cone Beam Computed Tomography in Dental Practice: An Endodontic Case Report
}

\author{
Monica Monea, Associate Prof., DMD, PhD
}

Department of Odontology and Oral Pathology, Faculty of Dental Medicine, University of Medicine and Pharmacy of Tîrgu Mureș, Romania

Kovacs Monika, Lecturer, DMD, PhD

Department of Odontology and Oral Pathology, Faculty of Dental Medicine,

University of Medicine and Pharmacy of Tîrgu Mureș, Romania

Nicoleta Suciu, postgraduate student

Department of European Research Projects, University of Medicine and

Pharmacy of Tîrgu Mureș, Romania

Pop Mihai, Lecturer, DMD, PhD

Department of Odontology and Oral Pathology, Faculty of Dental Medicine ,University of Medicine and Pharmacy of Tîrgu Mureș, Romania

doi: 10.19044/esj.2016.v12n33p11 URL:http://dx.doi.org/10.19044/esj.2016.v12n33p11

\begin{abstract}
We aim to present a case of obliterated root canal which was successfully treated in our dental faculty using these new techniques. Teeth with calcified root canals referred for endodontic treatment pose pa rticular diagnostic and treatment challenges. Usually these are intact traumatized anterior teeth without symptoms except of slight discoloration, without apical radiolucency. The thorough knowledge of endodontic anatomy and the use of modern equipment as the cone beam computed tomography (CBCT) and operating microscope are the basis for long term clinical and radiological success of these cases. The use of rotary endodontic instrumentation in conjunction with chelating agents as EDTA have been advocated for the endodontic instrumentation of narrow or obliterated root canals, where the apical dentin is usually more mineralized. CBCT images offered superior information in comparison to conventional radiographs but it must be taken into consideration only for difficult cases, due to higher radiation dose and costs.
\end{abstract}

Keywords: Obliterated root canals, CBCT, endodontic treatment, pulpal dystrophic calcification 


\section{Introduction}

Endodontic therapy of an obliterated root canal is considered to be a real challenge and therefore extensive knowledge regarding internal anatomy of the root canal system and accurate diagnostic techniques are essential to proper treatment and successful results. Root canal obliteration is usually a complication of dental trauma; it is defined as a deposition of hard tissue inside the pulp chamber and root canal, with important consequences on the vitality and the prognosis of the affected teeth [Carr et al 2010, Malthora et al 2013]. In the scientific literature it is also referred as calcific metamorphosis, diffuse or dystrophic calcification and can be recognized radiographically in a time interval between 3 months - 1 year [Krasner 2004, Chaman et al 2015]. Teeth with obliterated root canals are usually asymptomatic and the sensitivity tests are unreliable; there is a progressive reduction in the response to thermal and electrical tests, but the absence of positive reaction is not always an indication of pulp necrosis[McCabe et al 2012, Thomas et al 2014]. The purpose of our paper is to present a case of an obliterated central maxillary incisor which was referred to our department for non-surgical endodontic treatment.

\section{Case report}

We present the case of a male patient 35 years old referred to our clinic for endodontic treatment. The examination of tooth 11 revealed an asymptomatic discolored tooth with a history of trauma and a calcified root canal. The patient was informed and he signed an agreement for the proposed endodontic treatment and subsequent ceramic coronal restoration. An attempt had been made by the referring dentist to locate the root canal but as this proved to be impossible we decided to continue with an orthograde approach only after a CBCT investigation, in order to have a clear image of the entire endodontic system and surrounding structures. During the second visit, the access cavity was extended into the coronal third of the root but still the canal opening could not be located. A periapical radiograph confirmed the presence of via falsa in an oblique direction but without perforation and the presence of the canal only in the apical half of the root (Figure 1). 


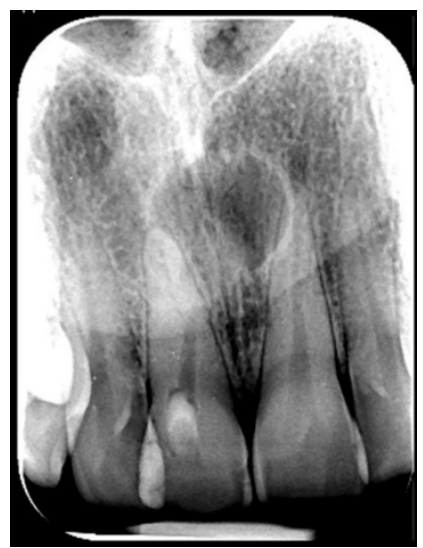

Figure 1. Conventional radiograph showing misdirected search of canal entrance, almost perforating the mesial wall of the root.

The CBCT scan revealed a somewhat palatally positioned canal entrance both in sagittal and horizontal sections (Figure 2 and 3).

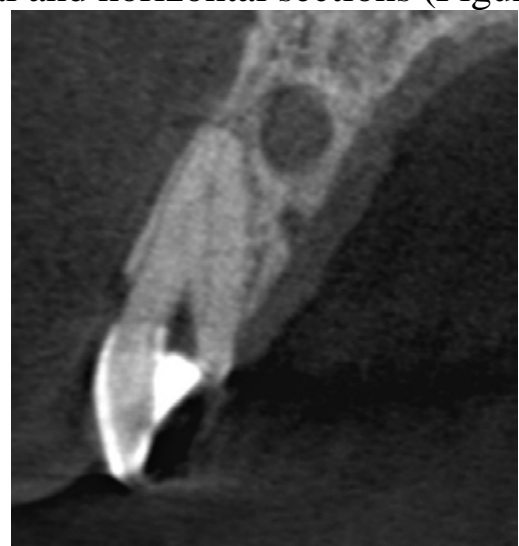

Figure 2. Screenshot from an axial view of the CT scan. The canal has an unusual, palatal position, making it difficult to locate the entrance.

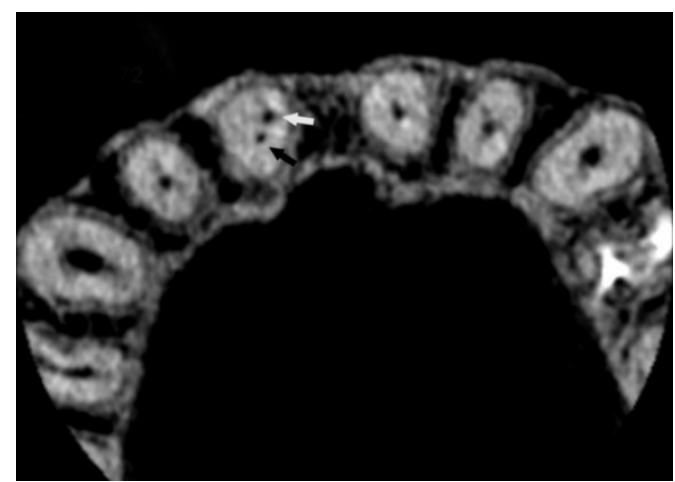

Figure 3. Horizontal scan demonstrating the position of the canal (black arrow) and the via falsa (white arrow). 
For the following clinical procedures an operating microscope was used in order to have magnification and proper illumination of the root canal. A long shank carbide bur at low speed was used to align the access cavity to the long axis of the tooth and the dentin was moistened with EDTA gel. The first instrument was a K-file \# 10 (Dentsply, Maillefer, Tulsa, USA) that established the glide-path to the working length and then rotary files MTwo (VDW GmbH, Munich, Germany) were used in the sequence indicated by the manufacturer as follows: $10 / .04 ; 15 / .05 ; 20 / .06$ and $25 / .06$. The root canal was irrigated with $25-30 \mathrm{ml}$ of sodium hypochlorite $5,25 \%$ heated at $50^{\circ} \mathrm{C}$. Both the root canal and the false canal were filled with 25/.06 tapered guttapercha cones and Adseal (Meta Biomed Co. Ltd., Korea) (Figure 4).

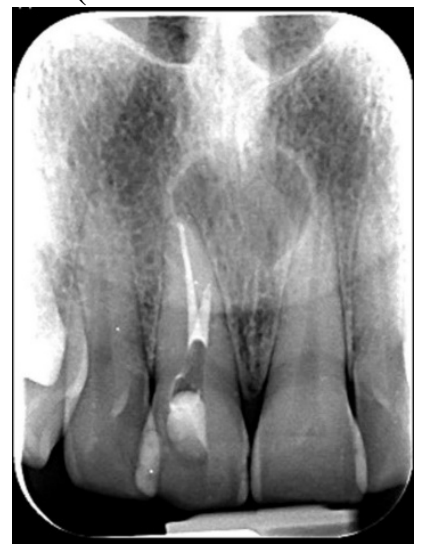

Figure 4. Periapical radiograph showing the filled canal and via falsa.

Glass ionomer cement (Fuji IX, GC Corp. Japan) was used as temporary coronal filling and the patient was advised not to delay for more than 3 weeks the final coronal restoration of the tooth.

\section{Discussion}

Root canal obliteration is seen in $4-24 \%$ of teeth after traumatic injuries, with radiographic absence of the root canal, as it was filled with mineralized tissue [Thomas et al 2014]. One of the most important steps in endodontic instrumentation is the preparation of the access cavity. In the case of anterior teeth, the access cavity is ideally made with a long shank round bur placed at an angle of $45^{\circ}$ with the long axes of the root, which will advance about 3-4 mm which will allow it to reach the pulp chamber. After that, the direction will become parallel to the tooth. This procedure is very safe if it is done under the magnification and improved lightning offered by the dental operating microscope [Carr et al 2010]. CBCT proved to be extremely efficient for identification of endodontic anatomy and visualization of missed root canals, in the evaluation of chronic periapical lesions and differential diagnosis between granulomas and cysts, which 
otherwise can be accomplished only by histological examination [Weber et al 2015]. It demonstrated certain superiority compared to 2-D radiographs. Conventional radiographs fail to identify the number of canals and missed anatomy can negatively influence the outcome of endodontic treatment. We agree to the recommendation made by Weissman et al in a recent study that the CBCT imaging technique is to be taken into consideration for those patients with persistent clinical symptoms and without a complete diagnosis based on the clinical and conventional radiographic examination [Weissman et al 2015]. Alongside to magnification provided by operating microscope, the CBCT will probably soon be considered the golden standard for treatment outcome evaluation for teeth with apical periodontitis. In an experimental study conducted by Weber et al the comparison made between conventional radiography and CBCT in the identification of missed canals, the percentage was $78 \%$ to $99 \%$ respectively [Weber et al 2015]. Furthermore, with CBCT it is possible to reconstruct the tooth morphology and its surrounding structures.

\section{Conclusion}

In the successful treatment of obliterated root canal, the importance of an adequate access cavity cannot be overemphasized, as it allows the identification of the initial canal. The use of gold standard instruments and imaging techniques, represented by the operating microscope and CBCT imaging system are prerequisite of modern endodontic therapy, which proved the efficacy in such difficult cases. CBCT examinations must be taken into consideration for cases in which the benefits of this investigation overcome the risks, offering additional information compared to conventional radiographic examination.

\section{Acknowledgements:}

The authors declare no conflict of interest. A written informed consent was obtained from the patient to publish all clinical data both in print and online.

\section{References:}

1. Carr G.B., Murgel C.A. The use of the operating microscope in endodontics. Dent Clin North Am; 2010 ,54: 191-214.

2. Malthora N., Mala K. Calcific metamorphosis: Literature review and clinical strategies. Dental Update; 2013, 40 (1):48-58.

3. Krasner P., Rankow H.J. Anatomy of the pulp chamber floor. J of Endodontics; 2004, 30: 5-16.

4. Chaman C., Garg P., Tyagi S.P., Singh V.P. Root canals- from concretion to patency. Saudi Endod J; 2015, 5: 13-19. 
5. McCabe P.S., Dummer P.M. Pulp canal obliteration: an endodontic diagnosis and treatment challenge. Int Endod J; 2012, 45 (2): 177197.

6. Thomas B., Chandak M., Patidar A., Deosarkar B., Kothari H. Calcified canals - A review. IOSR Journal of Dental and Medical Sciences; 2014, 13 (5); 38-43.

7. Weissman J., Johnson J.D., Anderson M., Hollender L., Huston T., Paranipe A., Patel S., Cohenca N. Association between the presence of Apical Periodontitis and clinical symptoms in Endodontic Patients using Cone-beam Computed Tomography and Periapical Radiographs. J Endod , 41 (11): 1824-9. doi: 10.1016/j.joen. 2015. 06. 004, Epub. 2015 sep5.

8. Weber M.T., Stratz N., Fleiner J., Schultze D., Hannig C. Possibilities and limits of imaging endodontic structures with CBCT. Swiss Dental Journal; 2015, 125 (3): 293-302. 